\title{
Minimax Polynomial Optimization by using Sum of Squares Relaxation and its Application to Robust Stability Analysis of Parameter-dependent Systems
}

\author{
Hiroyuki Ichihara and Eitaku Nobuyama
}

\begin{abstract}
This paper gives a sufficient condition for a robust control problem in terms of minimax polynomial optimization. A minimax problem is investigated by using sum of squares relaxation of parametric polynomial optimization problems. The sufficient condition can be exact from the Positivstellensatz. The minimax approach is adopted for robust stability analysis of parameter-dependent systems and an equivalent stability condition is also given from quadratic version of distinguished representation of positive polynomials. Finally, a numerical example is shown.
\end{abstract}

\section{INTRODUCTION}

Recently, a new method for solving a relaxed polynomial optimization problem (POP) by semidefinite programming (SDP) has been developed using sum of square (SOS) decomposition of positive semidefinite polynomials [1], [2]. It is possible to determine whether or not a polynomial function has SOS decomposition, and to compute global lower bounds for the polynomial function by solving SDP [3], [4]. Robust control using SOS optimization has been investigated in [5], [6], and positive polynomial representations via real algebraic geometry are summarized in [7].

The purpose of this paper is to investigate a robust control problem for parameter-dependent systems on the basis of Lagrangian dual of parametric-POPs. A robust stability problem is represented as a minimax-POP, and it is shown that the problem has a simple dual problem by using a distinguished representation of positive polynomials [8].

The following notation is used. $\mathbb{R}\left[x_{1}, \cdots, x_{n}\right]$ is the ring of real polynomials, which is abbreviated as $\mathbb{R}[x]$. The set of SOS polynomials is denoted by $\sum[x] . \mathbb{S}^{n}$ is the set of $n \times n$ symmetric matrices. $\mathbb{S}_{+}^{n}=\left\{X \in \mathbb{S}^{n} \mid X \succeq 0\right\}$. He $\{A\}$ means $A+A^{T} . A=\left\{a_{i j}\right\} \in \mathbb{R}^{n \times n}$ is vectorized as vec $A=$ $\left[a_{11}, a_{21}, \cdots, a_{n-1 n}, a_{n n}\right]^{T} \cdot d_{[N]}=\left(\begin{array}{c}n+N \\ N\end{array}\right), z_{[N]} \in \mathbb{R}^{d_{[N]}}$ is the monomial vector of $x$ whose maximum degree is $N$.

\section{LAGRANGIAN DUAL AND SOS RELAXATION}

Consider the minimax-POP

$$
\begin{array}{ll}
\max _{p} \min _{x} & f_{0}(x, p)=\sum_{\alpha} f_{0 \alpha}(p) x^{\alpha} \\
\text { subject to } & f_{i}(x) \geq 0, \quad i=1, \ldots, M,
\end{array}
$$

where $f_{0}(x, p), f_{i}(x) \in \mathbb{R}[x], f_{0 \alpha}(p)=f_{0 \alpha}(0)+c_{\alpha}^{T} p$, $x^{\alpha}=x_{1}^{\alpha_{1}} \cdots x_{n}^{\alpha_{n}}, \sum_{i=1}^{n} \alpha_{i} \leq 2 d, p \in \mathbb{R}^{m}$. Assume that $\mathcal{S}=\left\{x \in \mathbb{R}^{n} \mid f_{i}(x) \geq 0, i=1, \ldots, M\right\}$ is a compact

Department of Systems Innovation and Informatics, Kyushu Institute of Technology, 680-4 Kawazu Iizuka Fukuoka 820-8502, JAPAN. E-mail: \{ichihara,nobuyama\}@ces.kyutech.ac.jp semialgebraic set. Then a set $\mathcal{F}=\left\{x \in \mathbb{R}^{n} \mid f_{k}(x) f_{l}(x) \geq\right.$ $0,(1 \leq k<l \leq M), f_{k}(x) f_{l}(x) f_{m}(x) \geq 0,(1 \leq k<l<$ $m \leq M), \ldots\}$ is a redundant constraint set of the problem (1). Therefore, both the minimax-POP (1) and the problem

$$
\max _{p} \min _{x} \quad f_{0}(x, p) \quad \text { subject to } \quad x \in \mathcal{S} \cup \mathcal{F}
$$

have the same optimal value.

In order to construct a dual form of the parametric-POP part of (2), let us consider a function

$$
L(x, s, p)=f_{0}(x, p)-r(x)
$$

where $r(x) \in$ cone $\left\{f_{1}, \cdots, f_{M}\right\}$, cone $\left\{f_{1}, \cdots, f_{M}\right\}=$ $\left\{\sum_{i} s_{i}(x) \phi_{i}(x) \mid s_{i} \in \sum[x], \phi_{i} \in \operatorname{monoid}\left\{f_{1}, \cdots, f_{M}\right\}\right\}$, $\operatorname{monoid}\left\{f_{1}, \cdots, f_{M}\right\} \subset \mathbb{R}[x]$ is the set of all finite products of $f_{i}(x)$ together with 1 , and $s_{i}(x)$ 's are SOS multipliers. This is a generalization of standard Lagrangian functions of the parametric-POP (1) by using nonnegative scalar multipliers. Moreover, the Lagrangian function (3) has multipliers of many redundant inequality constraints. From this extension, both of the dual optimal value of parametric-POPs (1) and (2) are not always equivalent. Owing to this, the duality gap is expected to decrease. On the basis of the generalized Lagrangian dual and SOS relaxation, $p$ is required such that $f_{0}(x, p)$ is strictly positive on $\mathcal{S}$.

Theorem 1: If the optimal value of the SOS optimization problem (SOSOP)

$$
\begin{gathered}
\max _{\zeta \in \mathbb{R}, s_{i} \in \sum[x], p \in \mathbb{R}^{m}} \quad \zeta \\
\text { s.t. } L(x, s, p)-\zeta=s_{0}(x) \in \sum[x],
\end{gathered}
$$

is strictly positive, then the optimal value of the minimaxPOP (1) is also strictly positive.

The degree of $s_{0}(x)$ has to be fixed as $2 N$ in advance. Assume that $s_{0}(x)=z_{[N]}^{T} Q z_{[N]}$, then $s_{i}(x)=$ $z_{\left[N-w_{i}\right]}^{T} R_{i} z_{\left[N-w_{i}\right]}$, where $Q \in \mathbb{S}_{+}^{d_{[N]}}, R_{i} \in \mathbb{S}_{+}^{d_{\left[N-w_{i}\right]}}$ and $w_{i}=\lceil\{\operatorname{deg} \phi(x)\} / 2\rceil$. When $N$ is given, the SOSOP (4) can be solved by a SDP.

Lemma 1: Consider the SDP

$\max _{\zeta, Q, R_{i}, p} \quad \zeta$ subject to $\operatorname{trace} A_{\alpha} Q+\sum_{i} \operatorname{trace} B_{\alpha i} R_{i}-c_{\alpha}^{T} p+\zeta_{\alpha} \zeta=f_{0 \alpha}(0) \forall \alpha$, where $\zeta_{\alpha}$ is $1(\alpha=0)$, and $0(\alpha \neq 0)$. $A_{\alpha} \in \mathbb{S}^{d_{[N]}}$ and $B_{\alpha i} \in \mathbb{S}^{d_{\left[N-w_{i}\right]}}$ satisfy $z_{[N]} z_{[N]}^{T}=\sum_{\alpha} A_{\alpha} x^{\alpha}$ and $\phi_{i}(x) s_{i}(x)=\sum_{\alpha}\left(\operatorname{trace} B_{\alpha i} R_{i}\right) x^{\alpha}$, respectively. Then, the optimal value $\zeta$ is equivalent to the optimal value of the SOSOP (4). 
Theorem 1 with (3) is an instance of a stronger version of P-satz [9] which gives an exact representation of positive polynomials on the compact semialgebraic set $\mathcal{S}$. Thus, Lemma 1 can possibly be exact if all elements of cone are taken into account. However, high-order product terms make rapidly growth of computation [10]. Some kind of simple expression keeping its exactness without all products is desired.

\section{Application to Robust Stability AnAlysis of PARAMETER-DEPENDENT SYSTEMS}

Consider the parameter-dependent system

$$
\dot{y}(t)=A(\theta) y(t), \quad A(\theta)=A_{0}+\sum_{i=1}^{q} \theta_{i} A_{i}
$$

where $y \in \mathbb{R}^{a}, \theta \in \mathbb{R}^{q}, \Omega: \theta_{i} \in\left[\begin{array}{ll}\underline{\theta}_{i} & \bar{\theta}_{i}\end{array}\right](i=1, \ldots, q)$. Stability of this system is analyzed with a Lyapunov function with affine parameters $V(y, \theta)=y^{T} P(\theta) y, P(\theta)=$ $P_{0}+\sum_{i=1}^{q} \theta_{i} P_{i}$. The following condition is well-known.

Lemma 2 ([11]): For all $\theta \in \Omega$, if there exists $P_{0}, P_{1}, \ldots, P_{q} \in \mathbb{S}^{a}$ such that $\operatorname{He}\{P(\theta) A(\theta)\} \prec 0, P(\theta) \succ$ 0 , then the system (5) is exponentially stable.

Applying Theorem 1 to Lemma 2, a corollary is obtained.

Corollary 1: Given $\varepsilon>0$. Suppose that in SOSOP (4), $s_{i}(x)(i=0, \ldots, M), s_{j k}(x)(1 \leq j<k \leq$ $M), \cdots \in \sum[x], p=\left[\left(\operatorname{vec} P_{0}\right)^{T}, \quad \cdots, \quad\left(\operatorname{vec} P_{q}\right)^{T}\right]^{T} \in$ $\mathbb{R}^{a(a+1)(q+1) / 2}, x=\left[\begin{array}{ll}\phi^{T} & \theta^{T}\end{array}\right]^{T} \in \mathbb{R}^{2 a+q}$, and

$$
\begin{aligned}
f_{0}(x, p)=-\phi^{T}\left[\begin{array}{cc}
\operatorname{He}\{A(\theta) P(\theta)\} & 0 \\
0 & -P(\theta)+\varepsilon I
\end{array}\right] \phi \\
f_{i}(x)= \begin{cases}\bar{\theta}_{i}-\theta_{i} & (i=1, \ldots, q) \\
\theta_{i}-\bar{\theta}_{i} & (i=q+1, \ldots, 2 q) \\
\phi^{T} \phi-1 & (i=2 q+1) \\
1-\phi^{T} \phi & (i=2 q+2) .\end{cases}
\end{aligned}
$$

Then, if there exists strictly positive $\zeta$, the parameterdependent system (5) is exponentially stable.

Observing the above constraints, there are several affine constraints and two quadratic constraints. Moreover, highest degree's homogeneous part of one of the quadratic constraints, that is, $-\phi^{T} \phi$, is negative for every $x$. Then, the quadratic version of distinguished representations of positive polynomials is applicable to these constraints.

Lemma 3 ([8](Theorem 4.1)): Let $f_{i}(x) \in \mathbb{R}[x], \mathcal{S}$ is compact. Assume that for every $x \in \mathbb{R}^{n} \backslash\{0\}$ one of $\tilde{f}_{i}(x)$ is negative, where $\tilde{f}_{i}$ is the homogeneous part of $f_{i}$ of highest degree. Rearrange the sequence $f_{1}, \ldots, f_{M}$ in two sequences $f_{1}, \ldots, f_{v}$ of odd degree and $f_{v+1}, \ldots, f_{M}$ of even degree. Then every $f_{0}(x) \in \mathbb{R}[x]$ which is strictly positive on $\mathcal{S}$ has a representation

$$
\begin{aligned}
f_{0}(x)=s_{0}(x)+ & \sum_{i=1}^{M} s_{i}(x) f_{i}(x) \\
& +\sum_{1 \leq j<k \leq v} s_{j k}(x) f_{j}(x) f_{k}(x),
\end{aligned}
$$

where $s_{0}(x), s_{i}(x), s_{j k}(x) \in \sum[x]$.

Owing to this representation, it becomes clear that linear terms with all constraints and only second order product terms with affine constraints are needed. Thus, $L(x, s, p)=f_{0}(x, p)-\sum_{i=1}^{2 q+2} s_{i}(x) f_{i}(x)+$ $\sum_{1 \leq j<k \leq 2 q} s_{j k}(x) f_{j}(x) f_{k}(x)$ is adopted instead of (3).

\section{A NumericAl EXAMPLE}

In the system (5), suppose that $\bar{\theta}_{i}=-\underline{\theta}_{i}=1, \varepsilon=1.0$, $A_{0}=\left[\begin{array}{cc}0 & 1 \\ a_{0} & a_{0}\end{array}\right], \quad A_{1}=\left[\begin{array}{ll}0 & 0 \\ 1 & 0\end{array}\right], \quad A_{2}=\left[\begin{array}{ll}0 & 0 \\ 0 & 1\end{array}\right]$

and compute the maximum $a_{0}$ by using Corollary 1 as a feasible problem with the constraint $\zeta>0$ and dichotomy (scale width $1.0 \times 10^{-6}$ ). When $N=2, w_{i}=$ $1(i=1, \ldots, 6)$. The computation has been executed by using SOSTOOLS [12] and SeDuMi [13]. From eigenvalue analysis, the system is exponentially stable for $a_{0}<-1.0$. On the other hand, $\zeta=2.41 \times 10^{-5}(>0)$ and the maximum $a_{0}=-1.0000372(<-1.0)$ are obtained by Corollary 1. This result is close to the exact value. Note that it is infeasible with $a_{0}=-1.0$. This example shows how SOS relaxation technique gives a tight condition without relying on the convexity of the problem.

\section{CONCLUSIONS}

This paper gives a sufficient condition for robust stability of parameter-dependent systems in terms of a minimaxPOP. The minimax problem is investigated by using Lagrangian dual and sum of squares relaxation of parametricPOPs. A simpler condition than P-satz is obtained from the quadratic version of distinguished representation of positive polynomials. This approach could be applicable to controller design problems.

\section{REFERENCES}

[1] J. B. Lasserre, "Global optimization with polynomials and the problem of moments," SIAM J. of Optimization, vol. 11, pp. 796-817, 2001

[2] S. Kim, M. Kojima, and H. Waki, "Generalized lagrangian duals and sums of squares relaxation of square polynomial optimization problems," Tokyo Instituite of Technology, Tech. Rep. B-395, 2003.

[3] P. Parrilo, Ph.D. dissertation, California Institute of Technology, Pasadena, CA, may 2000.

[4] P. A. Parrilo and S. Lall, "Semidefinite programming relaxation and algebraic optimization in control," European Journal of Control, vol. 9, pp. 307-321, 2003.

[5] G. Chesi, A. Garulli, A. Tesi, and A. Vicino, "Robust stability of polytopic systems via polynomially parameter-dependent lyapunov functions," in 42th Conference on Decision and Control, Proc., 2003, pp. 4670-4681.

[6] P. A. Bliman, "A convex approach to robust stability for linear systems with uncertain scalar parameters," SIAM J. Control Optimization, vol. 42, pp. 2016-2042, 2004.

[7] A. Prestel and C. N. Delzell, Positive Polynomials. Berlin: Springer Monographs in Mathematics, 2001.

[8] T. Jacobi and A. Prestel, "Distinguished representations of strictly positive polynomials," Journal für die reine und angewandte Mathematik, vol. 532, pp. 223-235, 2001.

[9] K. Schmüdgen, "The K-moment problem for compact semi-algebraic sets," Mathematische Annalen, vol. 289, pp. 203-206, 1991.

[10] G. Stengel, "Complexity estimates for the schmüdgen positivstellensatz," J. Complexity, vol. 12, pp. 167-174, 1996.

[11] P. Gahinet, P. Apkarian, and M. Chilali, "Affine parameter-dependent lyapunov functions parametric uncertainty," IEEE Trans. Automat. Contr., vol. 41, pp. 436-442, 1996.

[12] S. Prajna, A. Papachristodoulou, P. Seiler, and P. A. Parrilo, SOSTOOLS: Sum of squares optimization toolbox for MATLAB, 2004. [Online]. Available: http://www.cds.caltech.edu/sostools and http://www.aut.ee.ethz.ch/ parrilo/sostools

[13] J. F. Strum, SeDuMi: Using sedumi 1.02, a matlab toolbox for optimization over symmetic cones (updated for version 1.05), 1998. [Online]. Available: http://fewcal.kub.nl/sturm/software/sedumi.html 